\title{
POSTERIOR DISPLACEMENT OSTEOTOMY OF THE CALCANEUS
}

\author{
G. P. Mitchell, Edinburgh, Scotland
}

In cases of established calcaneus after anterior poliomyelitis the deformity can be greatly reduced by combining an extensive plantar release with an oblique transverse osteotomy of the calcaneus that permits displacement upwards and backwards of the posterior weight-bearing part of the bone. The procedure greatly improves the mechanical advantage of subsequent tendon transplantations to the heel. Between 1956 and 1969 fifteen such osteotomies were carried out and the long-term results have been reviewed.

The deformity of calcaneus in association with paralysis of the triceps surae can of course be prevented in most cases by early tendon transplantation. The fully calcaneus-and have been unable to find any report of a precisely similar technique. The operation favoured by the Liverpool school (Dwyer 1964) involved the resection

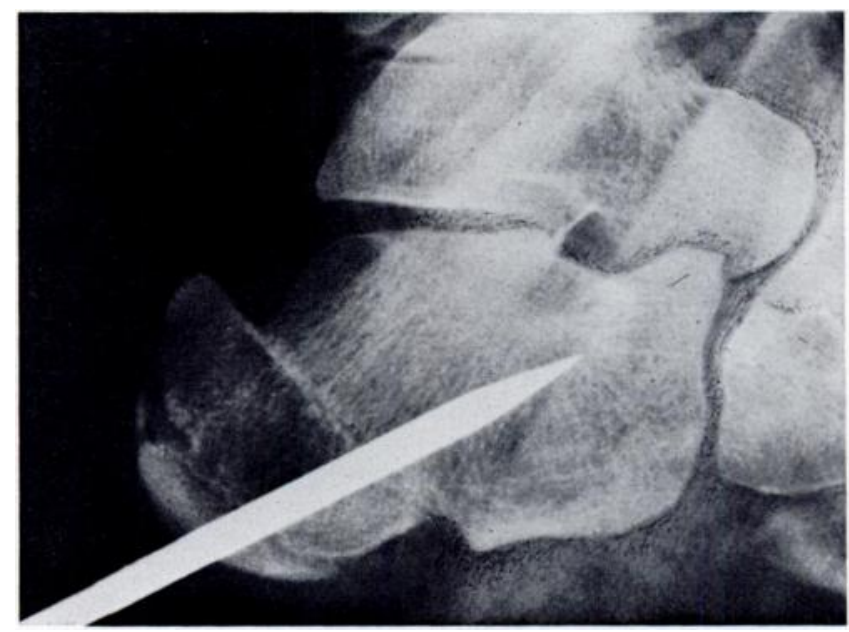

Fig. 1

A lateral radiograph taken at operation, showing the typical displacement of the osteotomy secured by a Steinmann pin.

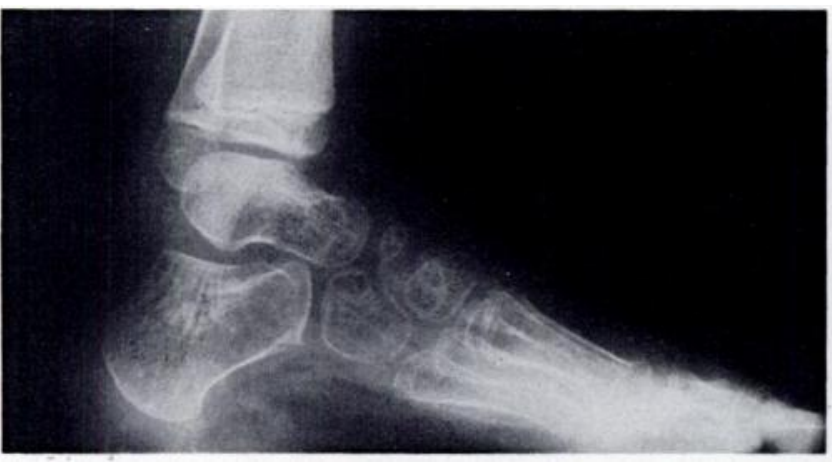

FIG. 2

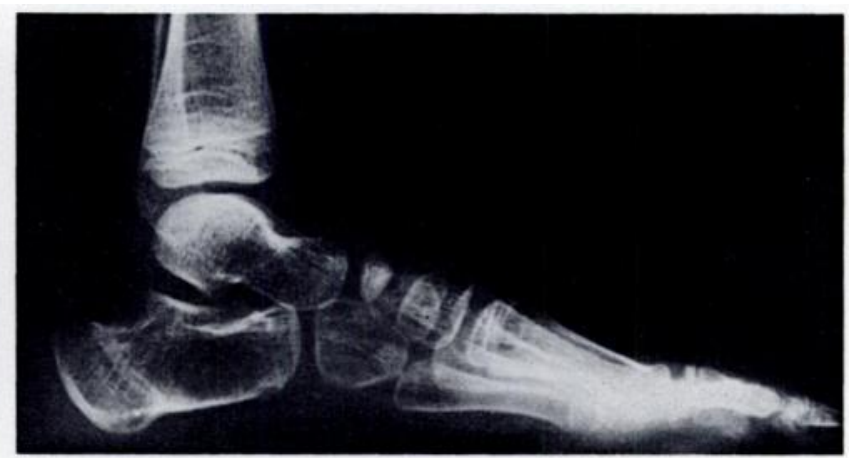

FIG. 3

Figure 2-A lateral radiograph showing a moderate degree of calcaneo-cavus. Figure 3-The same case eighteen months later showing elongation of the calcaneus and reduction of the deformity. No further procedure was required.

established deformity, however, demands correction by some kind of operation on the bony structure of the hindfoot, and the rather complicated Elmslie type of triple arthrodesis, with removal of a wedge based posteriorly from the subtalar region, has frequently been used for this purpose. Since 1956 we have used a much more simple procedure-posterior displacement osteotomy of the of a dorsal wedge from the calcaneus and hence shortening of the bone, which is a disadvantage.

\section{Clinical material}

Fifteen osteotomies were carried out in fifteen patients. The age at operation was between three and twenty-one years, the average being nine years.

George P. Mitchell, M.C., F.R.C.S.E., Princess Margaret Rose Orthopaedic Hospital, Fairmilehead, Edinburgh EH10 7ED, Scotland. 
Technique-An adequate plantar release is first performed through a short horizontal medial incision. The plantar muscles and fascia are stripped forwards from the calcaneal tubercles, the long and short plantar ligaments out by an oblique section inclined forwards and downwards just posterior to the peroneal tendons. The large posterior fragment is then displaced backwards and upwards, which will be possible only if the plantar

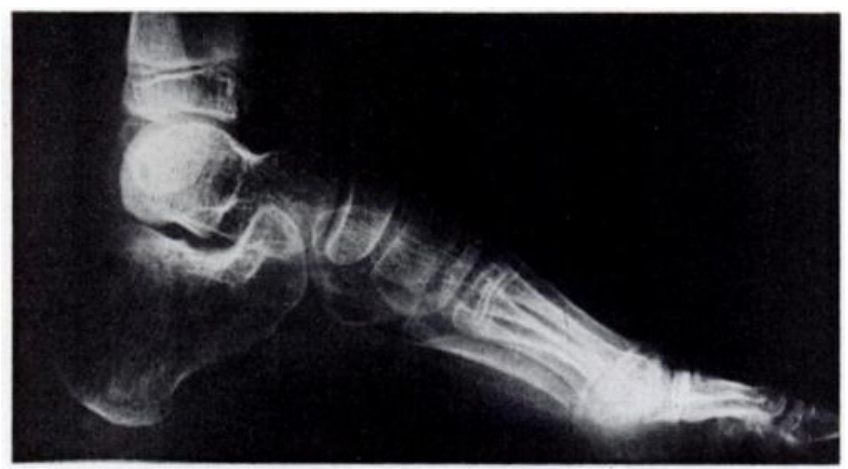

Fig. 4

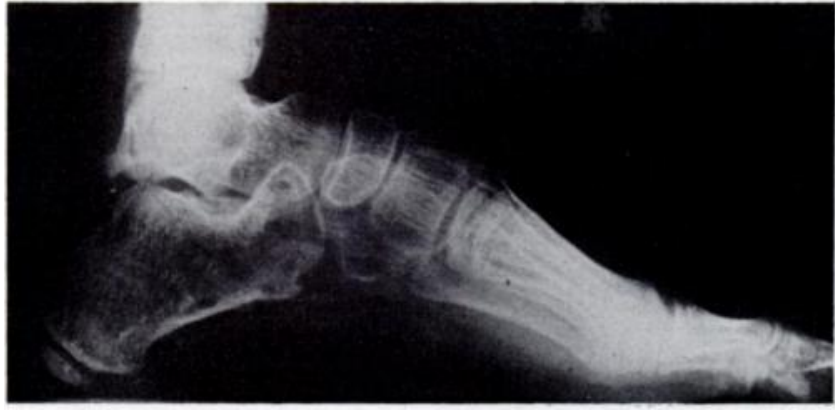

Fig. 5

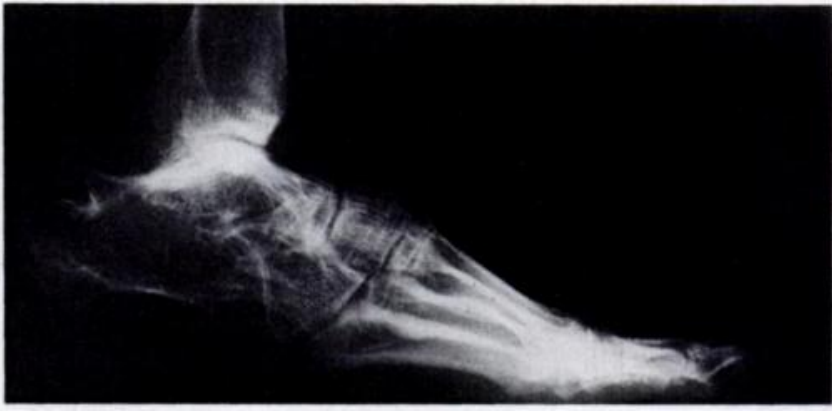

FIG. 6

Figure 4-A radiograph showing marked calcaneo-cavus. Figure 5-The same case two and a half years after osteotomy, showing partial correction of the deformity. An Elmslie posterior wedge type of triple arthrodesis was performed, followed by tendon transplantation. Figure 6-A radiograph of the same case twelve years later, taken with the limb bearing weight. The deformities have been fully corrected and the patient can now lift the heel clear of the ground.

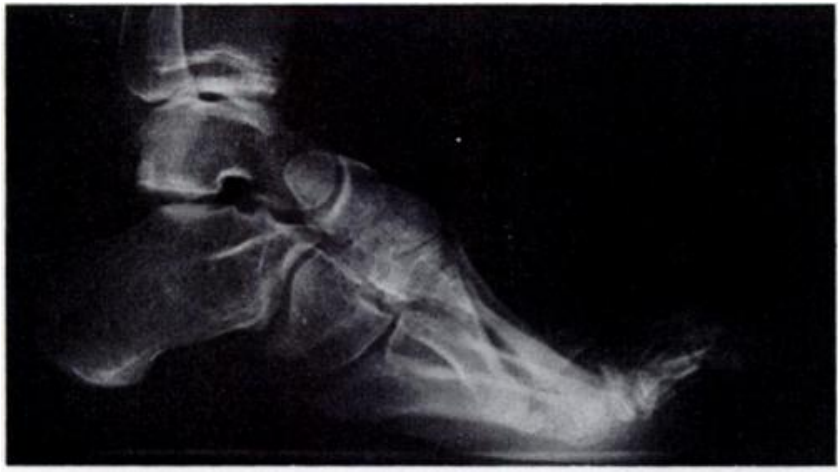

Fig. 7

A radiograph of a foot affected by a neurological disorder other than poliomyelitis, showing multiple deformities.

are divided, the calcaneo-cuboid and talo-navicular joints are opened by a sharp periosteal elevator and the foot is then forcibly manipulated by hand in order to obtain as much correction of cavus as the plantar release permits:

The lateral aspect of the calcaneus is now exposed through a curved lateral incision and the extent of the soft-tissue release is verified. The osteotomy is carried

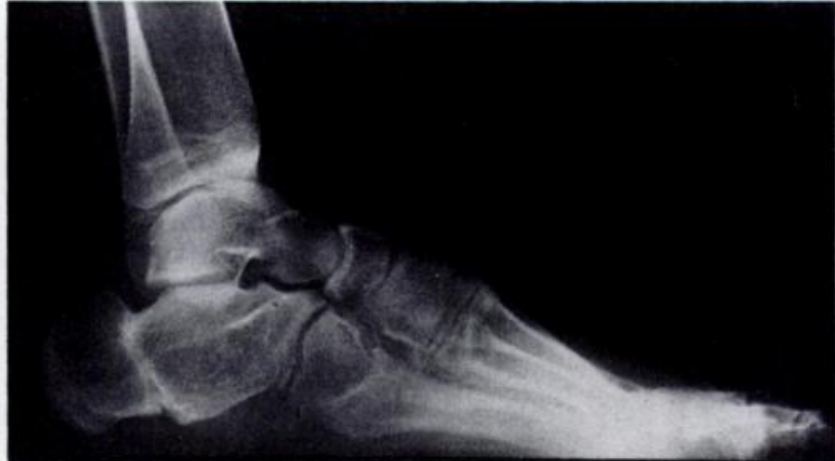

Fig. 8

The same case six months after calcaneal osteotomy with corrective arthrodesis of the toes and tendon transplantations.

structures have been freely released. The position of the fragment is secured by a Steinmann pin inserted through the skin of the heel and transfixing the osteotomy (Fig. 1).

A below-knee plaster is then applied incorporating the Steinmann pin, upward leverage on which aids reduction of the cavus deformity. After three or four weeks the pin is removed and a walking plaster applied. This is retained for a further month, by which time the 
osteotomy will have united. With this technique there have been no early complications such as dehiscence of wounds or infection of pin tracks. A typical case is shown in Figures 2 and 3.

\section{Later procedures}

Tendon transplantation-The indication for this procedure was severe weakness of triceps surae in the presence of one or more active long muscles suitable for transfer. The decision could be anticipated in some cases, but in others it was made after a period of observation of the effect of the osteotomy.

Five patients had transplantations. The cut end of the tendon was first threaded through the calcaneal tendon and then brought through a transverse tunnel in the calcaneus with the foot held in full equinus. In a case of multiple transplants the tendons were threaded through from opposite sides. The method demands firm tension on the tendons and seems to ensure good function of the transplanted long muscles.

The tendons thus transplanted were: both peronei (two cases); both peronei and tibialis posticus (one case); peroneus brevis, tibialis posticus and flexor longus hallucis (one case); and both peronei, tibialis posticus and flexor longus hallucis (one case).
Triple arthrodesis-Altogether eight feet required some variety of this operation at a later stage, but only four specifically for residual calcaneus deformity. Those four had a twostage Elmslie procedure with tendon transplantation (Figs. 4 to 6). Three feet had to be stabilised after tibial lengthening, which often leads to a valgus deformity. In our opinion triple arthrodesis would otherwise not have been necessary. Lastly, one foot required stabilisation for severe forefoot equinus.

Comment-The purpose of this paper is to suggest a simple method of reducing calcaneo-cavus deformity without resection of bone, and at the same time of lengthening the posterior lever arm of the calcaneus. When some power of plantar flexion remains, the operation may suffice by itself. Otherwise it can be regarded as a stage preparatory to further procedures such as tendon transplantation or some type of triple arthrodesis.

Due to the decline in poliomyelitis the operation has been carried out only three times since 1969 , in cases of severe pes cavus from other neurological disorders as a part of general reconstruction of the foot (Figs. 7 and 8).

\section{REFERENCE}

Dwyer, F. C. (1964) The relationship of variations in the size and inclination of the calcaneum to the shape and function of the whole foot. Annals of the Royal College of Surgeons of England, 34, 120-137. 